\title{
Concordancia Diagnóstica entre Sonohisterosalpingografía y la Laparoscopia en la Permeabilidad Tubárica de la Mujer Infértil Profamilia Medellín 1998
}

\author{
María Alejandra Palomino Zuluaga; Diana María Vallecilla Lukauskis; Oscar Alfredo Medina Tirado;Jorge Eduardo \\ Ramírez Vélez; Carlos Alberto Mejía Escobar; Jorge Alberto García Ortega; Jaime Gómez Díaz*
}

\section{RESUMEN}

OBJETIVO: Evaluar la concordancia diagnóstica de la sonohisterosalpingografia y la laparoscopia diagnóstica en la evaluación de la mujer infértil.

DISEÑO DEL ESTUDIO: Se realizó un estudio prospectivo comparando dos pruebas en la evaluación de la permeabilidad tubárica. La población fue de cincuenta mujeres entre 19 y 38 años con infertlilidad, se les realizó una sonohisterosalpingografia seguida de la laparoscopia y cromatoscopia. La prueba a estudio, la sonohisterosalpingografia, se realizaba entre los días 11 y 14 del ciclo, mediante la inserción de una sonda Foley \#8, atraves de la cual se infundía la solución salina sola o con aire y guiado con la ecografía transvaginal se visualizaba el paso a través de la cavidad uterina y las trompas. La laparoscopia y cromatoscopia se realizaba entre los dias 20 y 23 del ciclo.

RESULTADOS: la concordancia entre la sonohisterosalpingografia y la laparoscopia diagnóstica en la evaluación de la permeabilidad tubárica tuvo un acuerdo observado bruto del $92 \%$, un coeficiente kappa de 0.60 y p menor de 0.001 considerándose como una concordancia diagnóstica fuerte.

En las pacientes evaluadas se encontraron como hallazgos más frecuentes de la Sonohisterosalpingografía los ovarios poliquísticos con un $18 \%$ seguidos por los miomas intramurales con un $10 \%$.

CONCLUSIÓN: la sonohisterosalpingografía es una prueba útil para descartar patología ginecológica como anomalía de cavidad endometrial y obstrucción tubárica, y es una herramienta útil y de fácil acceso para el diagnóstico de otras patologías importantes en el manejo de la mujer infértil.

PALABRAS CLAVES: Infertilidad, Permeabilidad tubárica, Laparoscopia, Sonohisterosalpingografia.

\section{SUMMARY}

OBJECTIVE: The purpose of this study was to evaluate the use of the sonohysterosalpingography comparing with the chromolaparoscopy on the infertile women.

STUDY DESIGN: A prospective study was conducted comparing the sonohysterosalpingography with the chromolaparoscopy. Fiftytwo infertile women between 19-38 years old were included. Sonohysterosapingography was performed before the chromolaparoscopy on the preovulatory period ( days 11 to 14). Sterile saline solution and mixed with air bubbles was injected through a semirigid Foley catheter \#8 into the uterine cavity. Chromolaparoscopy was performed on the 23 th day of cycle.

RESULTS: the agreement between the sonohysterosalpingography and chromolaparoscopy was substantial with a kappa level of 0.6 .

The sonohysterosalpingography also allowed the diagnosis of Polycystic ovary (18\%). Followed by myomas (10\%).

CONCLUSIONS: the agreement between sonohysterosalpingography and chromolaparoscopy is substantial.

The technique evaluated offers additional anatomic information regarding the tubes, uterus and ovaries, important for the management of the infertile women.

KEY WORDS: Infertility, Tubal Patency, Laparoscopy, Sonohysterosalpingography.

* Especialización en Ginecología y Obstetricia. 


\section{Introducción}

La Infertilidad afecta del $10 \%$ al $15 \%$ de las parejas en edad fértil, con una incidencia anual de $1.6 / 1000$ parejas.(1)

Dentro de las diversas causas de infertilidad el $50 \%$ corresponde a alteraciones femeninas. En la evaluación de la mujer se identifican cuatro principales factores como son la disovulación que corresponde a un $40 \%$, la patología tubárica y pélvica (40\%), los problemas inusuales especialmente de carácter inmunológico y la infertilidad inexplicada.(1)

Para el estudio del factor tubo-peritoneal existen cinco técnicas que evalúan las lesiones de útero, trompa y peritoneo, siendo éstas la histerosalpingografía, laparoscopia, la histeroscopia, tubaroscopia y la sonohisterosalpingografía.

La Sonohisterosalpingografia tiene algunas ventajas para la paciente como son una evaluación de la cavidad uterina para el diagnóstico de anomalías intrauterinas como pólipos, miomas y malformaciones congénitas mullerianas.(2)

Otra ventaja de la técnica es que no hay exposición a radiación ionizante ni a materiales de contraste yodados como los utilizados en otros procedimientos; además es una técnica más segura y económica que la Laparoscopia diagnóstica y no requiere el empleo de anestésicos que aumentan los riesgos. $(3,4)$

En nuestro medio no se encontraron estudios comparativos de estas dos técnicas en la evaluación de la mujer infértil.

Por lo anteriormente descrito se pretendió evaluar los beneficios de la Sonohisterosalpingografía comparándola con la laparoscopia y cromatoscopìa en el estudio de la permeabilidad tubárica.

\section{Métodos}

Se tomó como población de estudio las mujeres que consultaron al servicio de Infertilidad de Profamilia de enero a diciembre de 1998 a las cuales se les programó una Laparoscopia diagnóstica para el estudio del factor tuboperitoneal, posterior a la realización de la sonohisterosalpingografía.

Se realizó un muestreo por conveniencia obteniéndose un tamaño de la muestra de cincuenta pacientes; se excluyeron pacientes que tenían estudios de evaluación de la permeabilidad tubárica previos y recientes, pacientes que no aceptaron entrar al estudio o que les quedara imposible asistir a la realización del examen en el día asignado y por último pacientes con alguna de las contraindicaciones para la realización de la sonohisterosalpingografía.

Los datos de interés fueron recopilados en un instrumento el cual fue diligenciado por los autores del trabajo.

Se programaba el procedimiento previa explicación y consentimiento informado.

Una vez realizado el contacto de la paciente en la consulta de infertilidad, se dió la cita de sonohis-terosalpingografia en la primera fase del ciclo menstrual, idealmente entre los días 11 y 14 del ciclo.

Se realizó una ecografía transvaginal inicial sin medio de contraste, durante la cual se examinó detalladamente la morfología de útero, ovarios y trompas.
El catéter o sonda de Foley, se introdujo con la ayuda de una pinza de curación a través del orificio cervical externo, a lo largo del canal cervical hasta la cavidad endometrial, donde se infló el balón con solución salina $2 \mathrm{cc}$.

Se llenó una jeringa estéril con 10 a 20 cc de solución salina. La jeringa se conectó al catéter o sonda y se realizó una infusión lenta del liquido mientras se observaba la imagen longitudinal del útero.

Se realizó una infusión lenta con 10 a 20 cc de solución salina sola o mezclada con aire mientras se tenía la imagen longitudinal del útero; el transductor es luego girado $90^{\circ}$ y se obtenía la imagen transversal de este para continuar evaluando la simetría y forma de la cavidad uterina.

En cada cuerno se identificó la unión uterotubárica y la porción intersticial de la trompa y se evaluó la existencia o no de los diferentes criterios de permeabilidad tubárica, como distensión de la trompa, flujo durante 5 segundos en la parte intersticial o líquido en el fondo de saco de douglas.

Después del procedimiento se dejó a la paciente en observación durante 20 minutos para vigilar la presencia o no de reacciones adversas tales como, hipotensión, mareo, dolor, sudoración, nausea entre otros. Para evitar complicaciones de carácter infeccioso se formuló profilaxis con cefalexina vía oral por 24 horas, además de antiinflamatorios no esteroides.

Se utilizó un ecógrafo marca Toshiba ECCCEE con transductor transvaginal multifrecuencia de 5-6-7.5 Mhz realizándose un promedio de tres procedimientos por sesión.

La información fue analizada mediante el programa SPSS versión 8.0; para evaluar la asociación entre los cruces de variables de interés se asumió una confiabilidad del $95 \%$.

La concordancia entre pruebas se realizó atraves del Kappa de Cohen.

\section{Resultados}

Se recolectaron inicialmente 52 pacientes de las cuales dos pacientes fueron excluidas ya que no se les pudo realizar la sonohisterosalpingografia por estenosis cervical intratable y por vaginismo respectivamente. Seis pacientes no se realizaron la laparoscopia y cromatoscopia, ya que tres de ellas se perdieron del estudio, una paciente se le programo miomectomia por el resultado de sonohisterosalpingografia y dos pacientes no aceptaron realizársela.

La edad promedio de las pacientes fue de 29 años, con una mínima de 19 años y una máxima de 38 años.

Mediante la sonohisterosalpingografia los resultados obtenidos fueron de un $85 \%$ de trompas permeables y un $15 \%$ de trompas obstruidas.(figura 1)

Con la laparoscopia el porcentaje de trompas permeables fue de $86.4 \%$ y de $13.6 \%$ para trompas obstruidas.(figura 2).

En las pacientes evaluadas se encontraron como hallazgos más frecuentes de la sonohisterosalpingografia los ovarios de aspecto poliquístico con un $18 \%$ (9 pacientes) seguidos por miomas intramurales con un $10 \%$ (5 pacientes).(figura 3 ). 


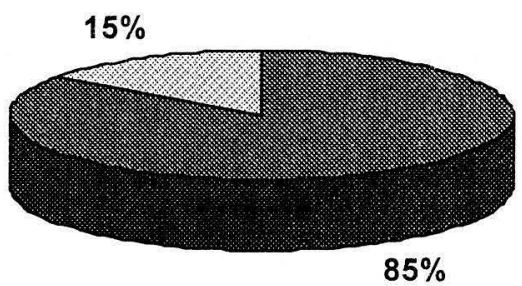

㘣 Trompas Permeables Trompas Obstruidas

Figura 1.

PROPORCION DE TROMPAS PERMEABLES SEGÚN LA SONOHISTEROSALPINGOGRAFÍA.

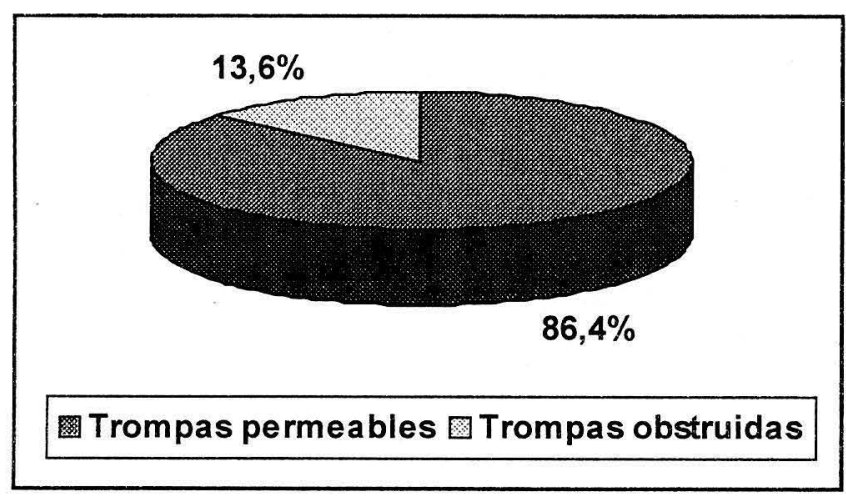

Figura 2.

PROPORCION DE TROMPAS PERMEABLES SEGÚN LA LAPAROSCOPIA

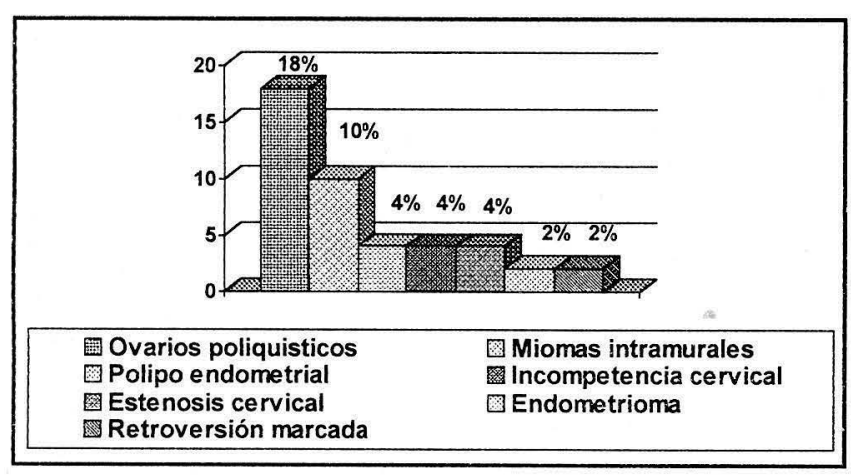

Figura 3.

\section{OTROS HALLAZGOS ENCONTRADOS EN LA SONOHISTEROSALPINGOGRAFÍA}

Uno de los parametros evaluados fue el del dolor durante la sonohisterosalpingografia, el cual fue calificado como leve, moderado o severo por la paciente; lo encontrado en el estudio fue que el $60 \%$ de las pacientes (31 pacientes) experimentó dolor leve, moderado un $31 \%$ (16 pacientes) y severo un $9 \%$ (5 pacientes).
El acuerdo observado bruto global fue de $92 \%$ y el acuerdo por azar de $80 \%$, para un coeficiente Kappa de 0.6 ; considerandose una concordancia diagnóstica fuerte.

\section{Discusión}

La sonohisterosalpingografía es un método de diagnostico nuevo en nuestro medio y hasta el momento no se había realizado un estudio comparativo entre esta técnica y la laparoscopia para evaluar la permeabilidad tubárica.

Entre los primeros estudios publicados en la literatura, que buscaban evaluar la permeabilidad tubárica mediante esta técnica se encuentra el de la doctora Tina S. Richman y col en 1984 (3), quienes evaluaron 34 pacientes infértiles mediante la sonohisterosalpingografia comparándolas con la histerosalpingografía.

Los resultados obtenidos demostraron una sensibilidad del $100 \%$ de la sonohisterosalpingografia para demostrar obstrucción tubárica y una especificidad de1 $96 \%$ para evaluar permeabilidad tubárica.

En nuestro estudio no se puede definir sensibilidad ni especificidad ya que no se contó con un grupo control de pacientes sanos; se calculó entonces el acuerdo observado bruto que fue de 0.92 , lo que hace referencia a que en un $92 \%$ de los casos hubo concordancia diagnóstica entre ambas técnicas. Como es importante evaluar que porcentaje de la concordancia se debió al azar se calculó el coefiente Kappa siendo éste de 0.60 , correspondiendo a una concordancia diagnóstica fuerte.

Lo anterior nos lleva a considerar la sonohisterosalpingografía como una prueba útil para descartar patología ginecológica (eg anomalía de cavidad endometrial y obstrucción tubárica).

Consideramos que esta prueba es inferior a la laparoscopia en pacientes a quienes se les sospeche patología tubárica por secuelas de enfermedad pélvica inflamatoria, endometriosis; es menos útil en el diagnóstico de entidades no inherentes a la cavidad uterina (adherencias, endometriosis, etc.).

Además, la sonohisterosalpingografía permitió el diagnóstico de otras patologías que son importantes para el manejo de la pareja infértil, como fueron nueve pacientes con ovarios poliquísticos, cuatro pacientes con miomatosis, una de las cuales se programó para miomectomia ya que interfería con la infertilidad, una paciente con un endometrioma, dos pacientes con incompetencia cervical, y dos pacientes con estenosis cervical que requirieron instrumentación antes de realizar la laparoscopia y una de ellas no fue posible realizar la , sonohisterosalpingografía, dos pacientes con pólipo endometrial, y una paciente con vaginismo siendo imposible realizar el examen en cuestión. Estos hallazgos confirman la utilidad de la prueba para el diagnóstico de otras patologías como anormalidades de la cavidad uterina, que permitirían realizar un abordaje y manejo inicial de la pareja infértil a mas bajo costo y con menos riesgos.

En cuanto a las reacciones adversas a la sonohisterosalpingografía se encontró que la tolerancia al dolor fue buena, catalogado como dolor leve el $62 \%$ de las pacientes, moderado $32 \%$ y severo solo el $6 \%$ de las pacientes. Tres pacientes presentaron reacción vagal al 
realizar el examen, cediendo con el manejo inicial, sin complicaciones posteriores, es de anotar que esta reacción fue observada en la realización de las primeras pruebas, relacionándose con el tiempo requerido, el cual fue mayor y la técnica estaba por estandarizase.

En conclusión, nuestro trabajo confirma la efectividad de la sonohisterosalpingografía respecto al diagnostico de la permeabilidad tubárica y se impone como un método de bajo costo con menos posibles reacciones de hipersensibilidad a medios de contraste utilizados en la histerosalpingografía.

Para evaluar la sensibilidad de esta prueba, sugerimos la realización de trabajos posteriores con un mayor número muestral y que comparen los resultados obtenidos con un grupo control de pacientes sanos.

\section{BIBLIOGRAFIA}

1. Speroff L,Glass R. Clinical Gynecologic Endocrinology and Infertility. Female Infertility 80, 1994.

2. Fleischer A, Manning F. Sonohystergraphy, sonosalpingography, and sonohysterosalpingography. Sonography in Obstetrics. Fifth edition. 1995: $932-967$

3. Richman T, Viscomi G. Fallopian Tubal Patency Assesed by ultrasound Following Fluid Inyection. Radiology 1984; 152: 507-510.
4. Laughead M, Stones L. Clinical utility of saline solution infusion sonohysterography in a primary care obstetric gynecologic practice. Am J Obstet Gynecol 1997; 176:13131318.

5. Tufecki E, Girit S. Evaluation of tubal patency by transvaginal sonosalpingography. Fertility and Sterility/1992; 57: $336-340$. 